\title{
Hypoxia-Induced PTTG3P Contributes to Colorectal Cancer Glycolysis and M2 Phenotype of Macrophage
}

\section{yue wang}

Liaoning Cancer Hospital \& Institute

guilin yu

Liaoning Cancer Hospital \& Institute

yiyang liu

Affiliated Hospital of Youjiang Medical University for Nationalities

longfei xie

University of California, Berkeley

jinnian ge

central hospital of Shenyang medical college

guohua zhao

Liaoning Cancer Hospital \& Institute

jie lin ( $\nabla$ gre031025@hotmail.com )

Liaoning Cancer Hospital \& Institute

\section{Research Article}

Keywords: Colorectal carcinoma, pseudogene, PTTG3P, YAP1, HIF1A

Posted Date: April 27th, 2021

DOI: https://doi.org/10.21203/rs.3.rs-426614/v1

License: (c) (1) This work is licensed under a Creative Commons Attribution 4.0 International License.

Read Full License

Version of Record: A version of this preprint was published at Bioscience Reports on July 1st, 2021. See the published version at https://doi.org/10.1042/BSR20210764. 


\title{
Hypoxia-induced PTTG3P contributes to colorectal cancer glycolysis and M2 phenotype of macrophage
}

Yue Wang ${ }^{[1]}$, Guilin $\mathrm{Yu}^{[1]}$, Yiyang Liu ${ }^{[2]}$, Longfei Xie ${ }^{[3]}$, Jinnian $\mathrm{Ge}^{[4]}$, Guohua Zhao ${ }^{[1]}$, Jie Lin ${ }^{[1]}$ [1] Department of General Surgery, Cancer Hospital of China Medical University, Liaoning Cancer Hospital \& Institute, 110042 Liaoning, P. R. China

[2] Department of Surgery, Affiliated Hospital of Youjiang Medical University for Nationalities, 533000, Guangxi Zhuang autonomous region, P. R. China

[3] Department of Physics and Integrative Biology, University of California, Berkeley, CA 94720

[4] The central hospital of Shenyang medical college, 110031 Liaoning, P. R. China

Lin Jie and Guohua Zhao are the co-corresponding authors.

Jie Lin, gre031025@hotmail.com, Cancer Hospital of China Medical University, Liaoning Cancer Hospital \& Institute, 110042 Liaoning, P. R. China

Guohua Zhao, glimmerbar@163.com, Cancer Hospital of China Medical University, Liaoning Cancer Hospital \& Institute, 110042 Liaoning, P. R. China

\begin{abstract}
Background Long noncoding RNAs (lncRNAs) play critical factors in tumor progression and are ectopically expressed in malignant tumors. Until now, lncRNA PTTG3P biological function in colorectal cancer (CRC) needs further to be clarified. Methods qRT-PCR was used to measure the PTTG3P level and CCK-8, glucose uptake, lactate assay, ATP assay, ECAR assay, and xenograft mice model were adopted to evaluate the glycolysis and proliferation, and macrophage polarization were determined in CRC cells. Xenograft experiments were utilized to analyze tumor growth. Results Ectopic expression of PTTG3P was involved in CRC and related to dismal prognosis. Through gain-of-function and loss-of-function approaches, PTTG3P enhanced cell proliferation and glycolysis through YAP1. Further, LDHA knockdown or glycolysis inhibitor (2-DG,3-BG) recovered PTTG3P-induced proliferation. And PTTG3P overexpression could facilitate M2 polarization of macrophages. Silenced PTTG3P decreased the level of inflammatory cytokines TNF- $\alpha$, IL-1 $\beta$, and IL-6, and low PTTG3P expression related with CD8+ T, NK, and TFH cell infiltration. Besides, HIF1A could increase PTTG3P expression by binding to the PTTG3P promoter region. Conclusions Hypoxia-induced PTTG3P contributes to glycolysis and M2 phenotype of macrophage, which proposes a novel approach for clinical treatment.
\end{abstract}

Keywords: Colorectal carcinoma, pseudogene, PTTG3P, YAP1, HIF1A 


\section{Background}

Colorectal cancer (CRC) remains one of the major triggers of deaths from malignant tumors. Globally more than 1 million people suffer CRC every year[1]. As of 2012, CRC is the fourth cause of cancer death after lung, stomach, and liver cancer[2]. Despite improvements in diagnosis and combined treatment, patients with CRC have an even worse prognosis, especially in advanced patients. Therefore, it is quite urgent to clarify the mechanism, even potential approaches for the therapeutic intervention of CRC.

Accumulating evidence has shown that pseudogene, a type of long noncoding RNA, exhibits pivotal functions. It is estimated that the human genome has more than 18,000 pseudogenes. And pseudogene has emerged as key regulators of important biological processes involved in the development of human cancers. For instance, increased CYP4Z1 expression promotes tumor angiogenesis and growth in breast cancer partly via PI3K/Akt and ERK1/2 activation[3]. Nanog regulates primitive hematopoiesis by directly repressing critical erythroid lineage specifiers[4]. PTENP1 can exert a growth-suppressive role by regulating cellular levels of PTEN[5]. LncRNA PTTG3P (pituitary tumor-transforming 3, pseudogene, NR_002734), located at chromosome 8q13.1, was first reported in the study of the human pituitary tumor transforming gene (hPTTG) family in 2000[6]. However, its biological function of the Warburg effect has yet to be illustrated in CRC.

Our data discovered that PTTG3P predicts poor prognosis in patients with CRC. Further study revealed that PTTG3P facilitates cell growth by regulating HIF1A/PTTG3P/YAP1 axis, and induces M2 polarization of macrophages.

\section{Materials and Methods}

\section{Clinical samples}

120 patients with CRC were enrolled from the Affiliated Hospital of Youjiang Medical University for nationalities, the central hospital of Shenyang medical hospital, and Cancer Hospital of China Medical University between March 2010 and November 2015. The including criteria were as follow: patients were with a definite pathological diagnosis; No patients were received with chemotherapy or radiotherapy before surgery. The tumor and paired non-tumor tissues were also collected after lesion excision with $30 \mathrm{mins}$ and stored in liquid nitrogen, then transferred to a $-80^{\circ} \mathrm{C}$ refrigerator. And the characteristics of cases were thoroughly noted. All of the CRC patients have signed informed consent before utilizing the clinical resources for investigation aims. The study was approved by the Ethics Committee of Youjiang Medical University for nationalities and Cancer Hospital of China Medical University.

\section{Cell lines culture}

Five human CRC cell lines (HT-29, SW620, HCT-8, SW480, and HCT116) and normal human intestinal epithelial cell lines (FHC, NCM460) were obtained from ATCC (Manassas, VA, USA). And cultured according to their instructions. All cells were cultured in an incubator according to their instructions at $37{ }^{\circ} \mathrm{C}$ and in a humidified atmosphere with $5 \% \mathrm{CO} 2$.

Total RNA isolation, qRT- PCR, and transfection 
The expression levels of RNA were calculated by the qRT-PCR system. Total RNA was extracted by TRIzol Reagent (Invitrogen), and $1 \mu \mathrm{g}$ of total RNA was reverse transcribed using the PrimeScript RT Reagent Kit (Perfect Real-Time; Takara). pcDNA3.1-PTTG3P, PTTG3P-containing lentiviral sequence vector (sh-PTTG3P) were purchased from GeneChem Corporation (Shanghai, China). CRC cells were transfected with plasmids in the presence of Lipofectamine 3000 (Invitrogen). After 48h of transfection, cells were gathered for further use in the following experiments. The gene expression quantity was calculated using the 2- $\Delta \Delta \mathrm{Ct}$ method. The oligonucleotides were transfected into CRC cells using Lipofectamine 2000 (Invitrogen, USA) and the transfection efficiency was confirmed by qRT-PCR. The detail is in Table S1, S2.

\section{Cell proliferation assay}

Cell viability assay was carried out to analyze cell proliferation. Cell viability was estimated using CCK8 (CK04, DOJINDO, Beijing, China), based on the manufacturer's instruction. Cells were seeded in 96-well culture plates. After incubation for the indicated time, a CCK-8 reagent (10 $\mu \mathrm{l})$ was added to each well. Cell viability was measured with a microplate reader for the absorbance at a wavelength of $450 \mathrm{~nm}$.

\section{Flow cytometry of apoptosis}

CRC cells in 6-well plates were rinsed in phosphate buffer saline (PBS), and then were trypsinized and resuspended in $100 \mu \mathrm{L}$ binding buffer added with $2.5 \mu \mathrm{L}$ of fluorescein isothiocyanate (FITC) conjugated Annexin V and $1 \mu \mathrm{L}$ of PI (Invitrogen). Fifteen minutes later, flow cytometry (BD Biosciences) was utilized for apoptotic cells.

\section{Glucose, lactate, adenosine triphosphate (ATP) levels, and extracellular acidification rate (ECAR)}

Glucose, lactate, adenosine triphosphate (ATP) levels, and extracellular acidification rate (ECAR). The levels of glucose and lactate were calculated with a Glucose Colorimetric Assay Kit (BioVision, CA) and a Lactate Assay Kit (BioVision, CA) in line with the instructions of the manufacturer. ATP level was tested using Cell Titer-Glo Luminescent Cell Viability Assay (Promega, Madison, MI). ECAR was detected using Seahorse XF 96 Extracellular Flux Analyzer (Agilent Technologies, Santa Clara, CA) according to the manufacturer's instructions.

\section{Chromatin Immunoprecipitation assay (ChIP)}

CRC cells $\left(2 \times 10^{6}\right)$ were used for ChIP assay according to the manufacturer's protocol of a ChIP assay kit (Millipore). The resulting precipitated DNA specimens were analyzed using PCR to amplify fractions of the PTTG3P promoter. The PCR products were resolved electrophoretically on a $2 \%$ agarose gel and visualized using ethidium bromide staining.

\section{Animal study}

HCT116 cells were transfected with sh-PTTG3P. $1 \times 107$ indicated cells were subcutaneously injected into 4-week-old male nude mice. Tumor volume measured every 5 days. 2-DG using in the study was injected into the abdominal cavity ( $1000 \mathrm{mg} / \mathrm{kg}$, injected into the abdominal cavity). Oxaliplatin using in the study was injected into the abdominal cavity ( $5 \mathrm{mg} / \mathrm{kg}$ twice per week). After 35 days, the twenty mice were sacrificed, and the tumor weight was measured. The animal study was carried out following 
the Guide for the Care and Use of Laboratory Animals of the NIH and the animal study was carried out in accordance with ARRIVE guidelines. This study had been approved by the Committee on the Ethics of Animal Experiments of Youjiang Medical University for nationalities and China medical university.

\section{Statistical analysis}

All the data were shown as the mean \pm standard deviation, at least three independent experiments. The difference between two independent groups was analyzed by a two-tailed Student's t-test, while multigroup comparison was made by ANOVA. Expression correlation between genes was analyzed by Pearson correlation analysis. Survival analysis was conducted using the Kaplan-Meier method and analyzed by the log-rank test. SPSS 22.0 (SPSS Inc., Chicago, IL, USA) was used to conduct statistical analyses, and differences were ensured when P-value was $<0.05$.

\section{Results}

\section{PTTG3P is highly expressed in CRC}

To evaluate potential IncRNAs involved in mediating CRC progression, we examined the lncRNA expression profile (GSE 84983) (Fig. S1a). Comparison between CRC tumor tissues and adjacent normal tissues, we focused on the upregulated lncRNAs (fold change $>5, \mathrm{P}<0.01$ ), for these IncRNAs might be oncogenes and therapeutic targets. LncRNA PTTG3P was one of the most upregulated and chosen for consideration (Fig. S1b). Then, we found that PTTG3P had rarely the ability to code proteins, using the open-reading frames (ORFs) Finder and conserved domain database. Moreover, five other different online metrics got the same conclusion (Table S3). Additionally, we identified no valid Kozak consensus sequence in PTTG3P[7], indicating that PTTG3P was a long noncoding RNA with no protein-coding potential. Then we explored the subcellular location of PTTG3P by using lncRNA subcellular localization predictor software (lncLocator, http://www.csbio.sjtu.edu.cn/bioinf/lncLocator/) (Fig.S1c), suggesting PTTG3P was mainly localized to the cytoplasm, and subcellular fractionation confirmed the prediction (Fig. S1d).

To verify the elevation of PTTG3P in CRC, we investigated the detailed annotative process of preclinical human cancer models via the Cancer Cell Line Encyclopedia (CCLE) (www.broadinstitute.org/ccle), indicating that PTTG3P was remarkably overexpressed in cell lines of CRC (Fig. 1a, 1b). Then, the cell lines of HT-29, SW620, HCT-8, SW480, HCT116, NCM460, and FHC were conducted for PTTG3P expression. As showed in Fig. 1c, the PTTG3P expression was exceedingly increased in HT-29, SW620, HCT-8, SW480, HCT116 cells, compared with NCM460 and FHC cells.

Further, we explored PTTG3P expression in a cohort of 120 paired and non-tumor tissues of CRC, the clinicopathologic characteristics are demonstrated in Table 1. Significantly, the PTTG3P level was overexpressed in CRC tissues compared to their counterparts (Fig. 1d, 1e), which was in accordance with the results of the TCGA database (Fig. 1f, 1g). Besides, high PTTG3P expression was observed in other malignant tumors (Fig. 1h). Also, our specimens confirmed PTTG3P overexpression in stomach adenocarcinoma (STAD), and esophageal squamous cell carcinoma (ESCA) (Fig. 1i,1j). Intriguingly, there was no actionable EGFR, VEGFR or RAS mutations, indicating that higher expressed PTTG3P may be driven by oncogenic event (Fig. S1e-1g). Altogether, these data revealed that PTTG3P was elevated in CRC and might be an oncogene. 


\section{High PTTG3P level correlates with poor prognosis}

To identify the connection between the level of PTTG3P and clinicopathologic features, we divided the cases into PTTG3P low-expression and high-expression groups based on the median expression.

Upregulated PTTG3P was positive linked with Tumor size $(\mathrm{P}=0.02)$ and Differentiation $(\mathrm{P}=0.01)$, but not with age $(\mathrm{P}=0.86)$, gender $(\mathrm{P}=0.74)$, tumor invasion depth $(\mathrm{P}=0.28)$, lymph node metastasis $(\mathrm{P}=0.09)$ or vessel invasion $(\mathrm{P}=0.06)$ (Table 1). Moreover, the PTTG3P expression in stage III-IV was higher than that in stage I-II tissues, and PTTG3P was expressed much more highly in advanced CRC samples than early CRC tissue (Fig. 2a). Additionally, Kaplan-Meier survival curves illustrated that patients with highly expressed PTTG3P had poorer survival time (Fig. 2b). Further, we determined the prognostic ability of PTTG3P in CRC. As shown in Table 2, univariate analyses suggested highly expressed PTTG3P was associated with a dramatic risk of death $(\mathrm{P}<0.01)$. Multivariate analysis demonstrated that PTTG3P expression was an independent prognostic factor $(\mathrm{P}<0.01)$. A model that incorporated the independent predictor was proposed as the nomogram (Fig.S2)

Subsequently, the ROC curve was carried out to evaluate the diagnostic capacity of PTTG3P in CRC tissues compared with normal counterparts, the area under the ROC curve (AUC) was 0.776 (95\% CI 0.733-0.819) (Fig. 2c). Thus, these data suggested that high expression of PTTG3P predicted a worse prognosis and may serve as a clinical biomarker for CRC patients.

\section{PTTG3P is caused by metabolic stress and promotes glycolysis and proliferation in CRC}

To investigate the biological function of PTTG3P, we transfected the PTTG3P overexpressed plasmids and shRNA targeting PTTG3P into HT-29 and HCT116 cells, respectively (Fig. 2d). By determining PTTG3P expression via gene set enrichment analysis (GSEA) the Cancer Genome Atlas (TCGA) profiles, we found that PTTG3P level was positively correlated with the glycolysis by affecting genes in glycolysis regulation (Fig. 2e). PTTG3P knockdown restrained the mRNA level of GLUT-1, ALDOA, PKM2, and LDHA, and the effect of sh-PTTG3P on glycolytic gene transcription could be rescued by PTTG3P re-expression (Fig. 2f).

And glucose deprivation is a well-known feature of solid tumors. Subsequently, we wonder whether PTTG3P participated in cell survival under metabolic stress, then we carried out several experiments with different glucose concentration and glycolysis inhibitor 2-deoxyglucose (2-DG) to make a condition of glucose deprivation. Obviously, PTTG3P expression was increased by glucose deprivation or 2-DG treatment in either dose-dependent or time-dependent manner (Fig. 2g, 2h). Thus, we elucidated that PTTG3P could play a crucial role in the progression of metabolic stress.

Next, we performed the glucose uptake analysis, ATP analysis, lactate production analysis, and discovered that sh-PTTG3P repressed these phenomena. In contrast, PTTG3P overexpression boosted glucose uptake (Fig. 3a), lactate production (Fig. 3b), and ATP accumulation (Fig. 3c). Additionally, we calculated the level of ECAR, sh-PTTG3P notably repressed glycolytic capacity and vice verse (Fig. 3d). Also, we found that silenced PTTG3P suppressed the proliferation, facilitated apoptosis of HCT116 cells, whereas upregulated PTTG3P increased the proliferation, inhibited apoptosis of HT-29 cells according to the CCK-8 assay and flow cytometry analysis (Fig. 3e,3f). In vivo, highly expressed PTTG3P efficiently increased the tumor growth (Fig. $3 \mathrm{~g}, 3 \mathrm{~h}$ ). We then explored whether glycolysis 
played a vital role in cell proliferation and tumor growth. Notably, the glycolic inhibitors 2-DG and 3-BP or depletion of LDHA, which catalyzed the final step of glycolysis, could partly abrogate cancer cell proliferation and tumor growth (Fig. 3i,3j,3k). Clinically, Oxaliplatin is used for the treatment of colorectal cancer. Previously, it is reported that suppression of glycolysis is an effective strategy to block cell proliferation and conquer drug resistance. As shown in Fig. 31,3m, PTTG3P depletion and Oxaliplatin played a synergistic role in emancipating tumor growth. As a taken, PTTG3P knockdown plus Oxaliplatin is a promising therapy for CRC.

\section{PTTG3P regulates Hippo signaling pathway in CRC}

In order to elucidate which pathway is involved in PTTG3P-mediated CRC progression, GSEA in the published TCGA CRC database was explored. And we suggested that PTTG3P expression was associated with the YAP1-activated gene signatures, indicating that Hippo signaling pathway might be involved (Fig. 4a). Then the hub genes in the Hippo pathway, including LATS1/2, MST1/2 and YAP1, and Hippo pathway target genes, such as CDX2, FOXM1, CTGF and CYR61, were checked in sh-PTTG3P HCT116 cells. Subsequently, PTTG3P knockdown impaired the level of YAP1, FOXM1 and CTGF (Fig. 4b). Moreover, silenced PTTG3P obviously decreased the enrichment of H3K27Ac at the YAP1 promoter, while that of H3K27me3 was increased (Fig. 4c). Next, we explored the TCGA database and drew the PTTG3P co-expression heat map. Then, we discovered that PTTG3P expression was correlated with genes in the Hippo pathway (YAP1, TEAD1-3), genes in the phenotype of proliferation (PCNA, MKI67, MCM2, MCM3, MCM5), gene in the phenotype of apoptosis (BAX, CASP1, CASP3, CASP10), gene in the phenotype of autophagy (ATG5), and genes in the phenotype of cell cycle (CDK1, CCDN1, CCND2, CCNB1), but not with MST1, an upstream factor of YAP1 (Fig. $4 d)$.

It is commonly acknowledged that YAP1, a crucial factor in the Hippo pathway, involves in cell proliferation and suppresses apoptotic genes, and YAP1 was highly expressed in CRC (Fig. S1h, S1i), and associated with advanced characteristics of CRC (Table S4). Further, YAP1 had a higher diagnostic value (AUC=0.793, 95\% CI:0.729-0.858) from the TCGA database (Fig. S1j). Additionally, we performed rescue assays in HT-29 cells. PTTG3P OE plus YAP1 KD could reverse the bioeffect of PTTG3P. Besides, we applied YAP1 inhibitors, CA3 (a novel specific YAP1 inhibitor) and verteporfin (an inhibitor of YAP1/TEAD interaction), got the same conclusion (Fig. 4e-4h). Intriguingly, the treatment of Hippo pathway inhibitor, XMU-MP-1 (inhibiting MST1/2), could not recover the effect of PTTG3P on proliferation, apoptosis and tumor growth (Fig. 4i4j).In brief, all the data uncovered that PTTG3P hedges the key factor MST1/2, while modulates YAP1 in the Hippo pathway to exhibit pivotal functions in CRC progression.

\section{PTTG3P promotes M2 phenotype Polarization of macrophage}

Tumor microenvironment (TME) is composed of tumor cells and surrounds non-tumor stromal cells, such as tumor associated macrophages(TAMs), carcinoma-associated fibroblasts and endothelial cells[8,9]. When the tumors occurs, tumor cells secrete numerous chemokines, recruit monocytes to permeate tumor tissues and then promote M2 phenotype. Subsequently, TAMs with M2 phenotype could in turn boost tumor growth, facilitate tumor cell metastasis and invasion, and suppress the immune system to promote tumor progression[10]. 
To elucidate whether PTTG3P play a role in M2 polarization, we explored the expression of PTTG3P, M1 markers (CD80, MCP-1, iNOS, and IL-6) and M2 markers (CD206 and MRC-2) in unpolarized macrophages, M1 macrophages treated by LPS/INF- $\gamma$ and M2 macrophages treated by IL4/IL13. Our data indicated that the expression of PTTG3P, CD206 and MRC-2 considerably were elevated in M2 macrophages, and then CD80, MCP-1, iNOS and IL-6 were increased in M1 macrophages (fig 5a), but PTTG3P was not increased in M1 macrophages (fig 5b). Next, we knocked down PTTG3P with siRNA after PMA (propidium monoazide) treatment for $24 \mathrm{~h}$, and utilized IL-4/ IL-13 to cause M2 phenotype. Interestingly, we found M2 markers (CD206 and MRC-2) were markedly diminished in si-PTTG3P group compared with si-NC group (Fig. 5c), while enforced expression of PTTG3P did the opposite (Fig. 5d). In addition, PTTG3P was correlated with CD206 (MRC1) and MRC2 from the TCGA-COAD database (Fig. 5e).

\section{PTTG3P is regulated by HIF1A under hypoxic conditions}

We next investigated the upstream factors which induced the elevated level of PTTG3P in CRC cells. Then, we treated CRC cells with hypoxia or $\mathrm{CoCl} 2$ (hypoxia chemical inducer) and found the PTTG3P expression in HT-29 cells was obviously increased as well as the elevation of HIF1A (Fig. 5f). While, depletion of HIF1A strikingly ameliorated PTTG3P expression in both normoxia and hypoxia condition (Fig. 5g, 5h). We also indicated that HIF1A and PTTG3P had a positive correlation (Fig. 5e, 5i). HIF1A was associated with advanced characteristics of CRC from the TCGA-COAD database (Table S5). Besides, HIF1A could partly rescue the effect of PTTG3P KD (Fig. 5j-51 ). In addition, ChIP analysis indicated that HIF1A enriched in the PTTG3P promoter region (Fig. 5m). Thus, the HIF1A/PTTG3P/YAP1 axis played a crucial role in CRC progression.

\section{PTTG3P plays vital functions in CRC immunology}

Cancer cells have high glucose uptake and glycolysis, resulting in a low level of glucose in the tumor, thus inhibiting the production of IFN-r by CD8+T cells in the tumor. The tumor immune environment broadly participates in different malignant tumors, including CRC. Recently, the treatment of CRC with immune checkpoint inhibitors (ICI) has provided a potential clinical treatment. Interestingly, our findings proposed that low PTTG3P expression relates with CD8+ T, NK and TFH cells infiltration in the microenvironment of CRC, not with Treg or macrophages infiltration, based on the TCGA database (Fig. 6a-6f). And the results of ELISA showed that the level of inflammatory cytokines TNF- $\alpha$, IL-1 $\beta$ and IL-6 were decreased with PTTG3P depletion (Fig. 6g). In addition, PTTG3P was correlated with TNF- $\alpha$, IL- $1 \beta$ and IL- 6 from the TCGA-COAD database (Fig. 5e).

\section{Discussion}

Pseudogenes are nonfunctional segments of DNA that resemble functional genes. Most arise as superfluous copies of functional genes, either directly by DNA duplication or indirectly by reverse transcription of an mRNA transcript. For instance, long noncoding RNA HK2P1 increased the lactate production and glucose uptake in endometrial stromal cells[11]. long non-coding RNA PTENP1 suppressed the PI3K/AKT signaling pathway and hindered the progression of HCC [12]. However, the role of PTTG3P in regulating CRC glycolysis has not been fully elucidated. Our study uncovered that PTTG3P boosted cell proliferation and glycolysis via the miR-1271-5p/PTTG3P/YAP1 axis. 
Our study verified that PTTG3P is highly expressed and has a potential diagnostic value, with an AUC of 0.776 (95\% CI 0.733-0.819) in CRC. Clinically, high PTTG3P expression considerably associates with tumor size and TNM stage as well as shorter survival time. These results confirmed that PTTG3P serves as a valuable prognostic biomarker and aids innovatively efficient therapies for CRC patients. Additionally, our findings stand in line with other research, Liu, et al.[13] reported that PTTG3P was remarkably upregulated in CRC tumor samples than that in normal samples. Zhou, et al.[14] revealed that PTTG3P is a valuable resource for identification in HCC progression and is useful for biomarker development. Weng, et al.[15] certified that PTTG3P facilitates cell proliferation, migration and invasion and might serve as a new promising strategy for gastric cancer. Recently, PTTG3P expression has a relationship with breast cancer[16] and pancreatic cancer[17]. Thus, the oncogenic role of PTTG3P in malignant tumors is strongly suggested.

Malignant tumors could undergo glycolysis at a higher speed than non-tumor tissue controls[18-20]. This phenomenon is known as the Warburg effect[21]. The Warburg hypothesis demonstrates that malignant tumor is fundamentally caused by mitochondrial metabolism disorder. Doherty JR, et al.[20] found that tumor lactate levels correlate with increased metastasis, tumor recurrence, and poor outcome. And targeting lactate metabolism is a prospective method for cancer therapeutics. Furthermore, cancer cells with a high level of glycolysis and acid resistance have an energetic growth advantage, which facilitates unrestrained proliferation and invasion. In our study, we explored gain and loss-of-function approaches in HT-29 and HCT116 cells and found PTTG3P ablation resulted in the inhibition of CRC cell glycolysis by regulating numerous genes linked with metabolic pathways, whereas the opposite outcome was observed after enforced expression of PTTG3P. Nowadays, a ketogenic diet was used to constrain glycolysis to starve cancer cells, adjusting mitochondrial metabolism[22]. Here, we also proposed that the biological mechanism of PTTG3P on boosting cell proliferation might resist apoptosis.

Hippo signaling pathway has become increasingly important in human cancer[23], the key regulator YAP1 has been certified to be upregulated in breast cancer, colorectal cancer, and liver cancer[24], and YAP1 could promote cell growth[25-27] and inhibit apoptosis[28]. Clinically, YAP1 could be a target for the development of cancer drugs[29]. Yi, et al. [30] suggested that inhibiting TEAD-YAP1 interactions or block the binding function of WW domains is a pharmacologically viable strategy against the YAP1 oncoprotein. In our presented study, we discovered that PTTG3P activates Hippo signaling pathway by promoting YPA1, FOXM1 and CTGF, not MST1/2, and rescue assay consolidates this by using the Hippo pathway inhibitor, XMU-MP-1 (inhibiting MST1/2).

These years, cancer has been considered to be a complex system including the tumor microenvironment (TME). Tumor-associated macrophages (TAMs) are the most common immune-related stromal cells in the TME, and communication between cancer cells and TAMs is crucial for the progression of epithelial ovarian cancer (EOC)[31]. Recently, a great amount of studies elucidated that TAMs has the property of M2 macrophages that are related to cancer progression. Our finding suggested that PTTG3P expression was elevated in M2 macrophages, not in M1 macrophages or unpolarized macrophages. Next, enforced PTTG3P markedly increased the M2 macrophages markers (CD206 and MRC-2), while PTTG3P depletion attenuated these markers. Hence, PTTG3P might play a vital role in $\mathrm{M} 2$ polarization progression. 
During cancer progression, tumor cells acquire comprehensive metabolic reprogramming, and tissue hypoxia is a prominent feature of solid tumors leading to cell metabolism adaptive changes.

Hypoxia-inducible factor- $1 \alpha$ (HIF1A) is a key oxygen-regulated transcriptional activator, playing a fundamental role in the adaptation of tumor cells to hypoxia by upregulating the transcription of target genes related to multiple biological processes, including cell survival, proliferation, angiogenesis and anti-apoptosis[32, 33]. In the microenvironment of oxygen-glucose shortage, HIF1A is a major factor in cancer survival. Zhou et al.[34] proposed that HIF1A activated IncRNA RAET1K modulated glycolysis in hepatocellular carcinoma cells via miR-100-5p. Tiwari, et al.[35] discovered that HIF1A might act as a tumor suppressor by preventing the expression of PPP1R1B and subsequent degradation of the $\mathrm{p} 53$ protein in pancreatic cancer cells. Our study indicated that HIF1A increased PTTG3P expression at the transcription level and enriched in the promoter region of PTTG3P, and could partly rescue the bioeffect of PTTG3P.

Last, our findings proposed that low PTTG3P expression relates with CD8+ T, NK and TFH cells infiltration in the microenvironment of CRC, not with Treg or macrophages infiltration from the TCGA database. And we found that a decreased TNF- $\alpha$, IL-1 $\beta$ and IL- 6 level was accompanied by ablation of PTTG3P.

\section{Conclusion}

In summary, we found hypoxia-induced PTTG3P was an oncogene in CRC, and higher PTTG3P expression was related to a dismal prognosis. PTTG3P might facilitate cell proliferation, glycolysis and M2 phenotype of macrophage. Thus PTTG3P plus HIF1A should be together adopted as critical targets for the prevention and therapy of CRC, illuminating some light on the understanding of the lncRNAs in CRC progression.

\section{Data Availability}

The datasets used and analyzed in the current study are available from the corresponding author on reasonable request.

\section{Competing Interests}

The authors declare that there are no competing interests associated with the manuscript.

\section{Funding}

Supported by Natural Science Foundation of Liaoning Province of China.

\section{Author Contributions}

The work presented here was carried out in collaboration with all authors. Yue wang and guohua Zhao contributed to the conception of the study; Yang zheng and Guilin yu contributed significantly to the experiment. Yiyang Liu, Longfei xie and Jinnian Ge performed the data analyses and wrote the manuscript;

\section{Statement of Ethics}

Patients have given their written informed consent in our study. And ethics committee of Affiliated Hospital of Youjiang Medical University for Nationalities and Liaoning cancer hospital approved the 
study protocol. The study protocol conforms to the ethical guidelines of the 1975 Declaration of Helsinki.

\section{Consent to Publication}

All the authors agreed to publish the manuscript.

\section{Acknowledgment}

We appreciate all the participants who provide supports for our research.

\section{Reference}

[1] Cunningham D, Atkin W, Lenz HJ, et al. Colorectal cancer. Lancet.2010, 375 (9719): 1030-1047.

[2] Adelstein BA, Macaskill P, Chan SF, et al. Most bowel cancer symptoms do not indicate colorectal cancer and polyps: a systematic review. BMC Gastroenterology. 2011,11: 65.

[3] Michael A Rieger, Reinhard Ebner, David R Bell, et al. Identification of a novel mammary-restricted cytochrome P450, CYP4Z1, with overexpression in breast carcinoma. Cancer Res.2004, 64, 2357-2364.

[4] Wei Yu, Hongyan Chai, Ying Li, et al.Increased expression of CYP4Z1 promotes tumor angiogenesis and growth in human breast cancer. Toxicol Appl Pharmacol. 2012,264(1): 73-83. [5] Laura Poliseno, Leonardo Salmena, Jiangwen Zhang, et al. A coding-independent function of gene and pseudogene mRNAs regulates tumor biology. Nature. 2010,24; 465(7301): 1033-1038.

[6] Chen L, Puri R, Lefkowitz EJ, Kakar SS. Identification of the human pituitary tumor transforming gene (hPTTG) family: molecular structure, expression, and chromosomal localization. Gene. 2000;248:41-50.

[7] Kozak M. Point mutations define a sequence flanking the AUG initiator codon that modulates translation by eukaryotic ribosomes. Cell. 1986;44: 283-292.

[8] Noy R, Pollard JW. Tumor-associated macrophages: from mechanisms to therapy. Immunity. 2014;41:49-61.

[9] Kerkar SP, Restifo NP. Cellular constituents of immune escape within the tumor microenvironment. Cancer Res. 2012;72:3125-3130.

[10]Zhe Ge, Shuzhe Ding. The Crosstalk Between Tumor-Associated Macrophages (TAMs) and Tumor Cells and the Corresponding Targeted Therapy.Front Oncol. 2020; 10: 590941.

[11] Hong Lv, Jing Tong, Jieqiong Yang, et al. Dysregulated Pseudogene HK2P1 May Contribute to Preeclampsia as a Competing Endogenous RNA for Hexokinase 2 by Impairing Decidualization. Hypertension. 2018,71(4):648-658.

[12] Chiu-Ling Chen, Yen-Wen Tseng, Jaw-Ching Wu, et al. Suppression of hepatocellular carcinoma by baculovirus-mediated expression of long non-coding RNA PTENP1 and MicroRNA regulation. Biomaterials. 2015,44:71-81.

[13] Liu N, Dou L, Zhang X. LncRNA PTTG3P Sponge Absorbs microRNA-155-5P to Promote Metastasis of Colorectal Cancer. 2020,(9)13:5283-5291.

[14] Zhou Q, Zhang W, Wang Z, et al. Long non-coding RNA PTTG3P functions as an oncogene by sponging miR-383 and up-regulating CCND1 and PARP2 in hepatocellular carcinoma. BMC Cancer. 2019, 24;19(1):731.

[15] Weng W, Ni S, Wang Y, et al. PTTG3P promotes gastric tumor cell proliferation and invasion and is an indicator of poor prognosis. J Cell Mol Med. 2017,21(12):3360-3371. 
[16] Lou W, Ding B, Fan W. High Expression of Pseudogene PTTG3P Indicates a Poor Prognosis in Human Breast Cancer. Mol Ther Oncolytics. 2019,27;14:15-26.

[17] Liu W, Tang J, Zhang H, et al. A novel lncRNA PTTG3P/miR-132/212-3p/FoxM1 feedback loop facilitates tumorigenesis and metastasis of pancreatic cancer. Cell Death Discov. 2020,30;6(1):136.

[18] GATENBY R A, GILLIES R J. Why do cancers have high aerobic glycolysis? Nat Rev Cancer, 2004, 4(11): 891-899.

[19] OU J, MIAO H, MA Y, et al. Loss of abhd5 promotes colorectal tumor development and progression by inducing aerobic glycolysis and epithelial-mesenchymal transition. Cell Rep, 2014, 9(5): 1798-1811.

[20] DOHERTY J R, CLEVELAND J L. Targeting lactate metabolism for cancer therapeutics. J Clin Invest, 2013, 123(9): 3685-3692.

[21] WARBURG O. On the origin of cancer cells. Science, 1956, 123(3191): 309-314.

[22] SCHWARTZ L, SEYFRIED T, ALFAROUK KO, et al. Out of Warburg effect: An effective cancer treatment targeting the tumor-specific metabolism and dysregulated $\mathrm{pH}$. Semin Cancer Biol, 2017, 43:134-4138.

[23] Saucedo LJ, Edgar BA. Filling out the Hippo pathway. Nat Rev Mol Cell Biol. 2007,8(8):613-621.

[24] Zender L, Spector MS, Xue W, et al. Identification and validation of oncogenes in liver cancer using an integrative oncogenomic approach.Cell. 2006,30; 125(7): 1253-1267.

[25] Shimomura T, Miyamura N, Hata S, et al. The PDZ-binding motif of Yes-associated protein is required for its co-activation of TEAD-mediated CTGF transcription and oncogenic cell transforming activity. Biochemical and Biophysical Research Communications. 2014,443(3): 917-923.

[26] Harvey KF, Zhang X, Thomas DM. The Hippo pathway and human cancer. Nature Reviews. Cancer. 2013,13 (4): 246-257.

[27] Johnson R, Halder G. The two faces of Hippo: targeting the Hippo pathway for regenerative medicine and cancer treatment. Nature Reviews. Drug Discovery. 2014,13 (1): 63-79.

[28] Zhao B, Kim J, Ye X, et al. Both TEAD-binding and WW domains are required for the growth stimulation and oncogenic transformation activity of yes-associated protein. Cancer Research. 2009, 69 (3): 1089-1098.

[29] Sudol M, Shields DC, Farooq A. Structures of YAP protein domains reveal promising targets for development of new cancer drugs. Seminars in Cell \& Developmental Biology. 2012, 23 (7): 827-833.

[30] Liu-Chittenden Y, Huang B, Shim JS, et al. Genetic and pharmacological disruption of the TEAD-YAP complex suppresses the oncogenic activity of YAP. Genes \& Development. 2012, 26 (12): 1300-1305.

[31] Chen X, Zhou J, Li X, et al. Exosomes derived from hypoxic epithelial ovarian cancer cells deliver microRNAs to macrophages and elicit a tumor-promoted phenotype. Cancer Lett. 2018;435:80-91.

[32] Deng SJ, Chen HY, Ye Z, et al. Hypoxia-induced LncRNA-BX111 promotes metastasis and progression of pancreatic cancer through regulating ZEB1 transcription. Oncogene.

2018;37:5811-5828.

[33] Wang Y, Liu X, Zhang H, et al. Hypoxia-inducible IncRNA-AK058003 promotes gastric cancer metastasis by targeting gamma-synuclein. Neoplasia. 2014;16:1094-1106.

[34] Yufan Zhou, Yun Huang, Kuan Hu, et al. HIF1A activates the transcription of IncRNA RAET1K to modulate hypoxia-induced glycolysis in hepatocellular carcinoma cells via miR-100-5p. Cell Death Dis. 2020, 11(3):176. 
[35] Ashutosh Tiwari, Kojiro Tashiro, Ajay Dixit, et al. Loss of HIF1A From Pancreatic Cancer Cells Increases Expression of PPP1R1B and Degradation of p53 to Promote Invasion and Metastasis. Gastroenterology. 2020, 59(5):1882-1897.

\section{Figure legend}

Figure 1 PTTG3P is upregulated in CRC tissues, and cell lines.

(A, B) Exploring PTTG3P expression in CRC cell lines by assembling the Cancer Cell Line Encyclopedia (CCLE) (www.broadinstitute.org/ccle).

(C) The expression profiles of PTTG3P in HT-29, SW620, HCT-8, SW480, HCT116, NCM460 and FHC was detected with qRT-PCR.

(D, E) qRT-PCR analysis was used to evaluate PTTG3P expression in 120 paired tumor and paired adjacent non-tumor tissues.

$(\mathrm{F}, \mathrm{G})$ High PTTG3P expression was observed in the TCGA database of CRC $(\mathrm{n}=521)$.

(H) High PTTG3P expression was observed in the TCGA database of malignant tumors.

$(\mathrm{I}, \mathrm{J})$ High PTTG3P expression was observed in STAD and ESCA $(\mathrm{n}=20)$.

$* \mathrm{P}<0.05,{ }^{* *} \mathrm{P}<0.01, * * * \mathrm{P}<0.001$

Figure 2 Overexpressed PTTG3P correlates with poor prognosis in CRC.

(A) The expression of PTTG3P was checked in different clinical stages of CRC tissues

(B) PTTG3P expression and survival predicted poor prognosis of OS in a cohort of 120 paired cases.

(C) ROC curve of PTTG3P.

(D) Short hairpin RNA (shRNA) targeting PTTG3P and PTTG3P overexpressed plasmids were transfected into HCT116 and HT-29 cells.

(E) GSEA plot showing that PTTG3P expression positively correlated with glycolysis-activated gene

signatures (REACTOME GLYCOLYSIS).

(F) Analysis of glycolic gene expression in PTTG3P knockdown or re-expressed PTTG3P.

(G) qPCR analysis showed that PTTG3P was upregulated under low glucose culture conditions compared to normal glucose in a dose-dependent manner and time-dependent manner.

(H) QPCR analysis showed that PTTG3P was increased after 2-DG treatment in dose-dependent manner and in time-dependent manner.

$* \mathrm{P}<0.05,{ }^{* *} \mathrm{P}<0.01,{ }^{* * *} \mathrm{P}<0.001$

Figure 3 PTTG3P promotes the glycolysis and proliferation of CRC.

(A) Glucose uptake analysis, (B) Lactate production analysis, (C) ATP analysis explored the glucose uptake, lactate production, and ATP accumulation level, with sh-PTTG3P or overexpressed PTTG3P in HCT 116 or HT-29 cells.

(D) Extracellular acidification rate (ECAR) analysis tested the glycolytic capacity in CRC cells with sh-PTTG3P or overexpressed PTTG3P in HCT116 or HT-29 cells.

(E) CCK8 assay detected the proliferation of HCT116 and HT-29 cells transfected with sh-PTTG3P or overexpressed PTTG3P.

(F) Flow cytometry assays revealed that PTTG3P affected cell apoptosis.

(G) Tumor volume and $(\mathrm{H})$ weight were measured in vivo when injected with overexpressed PTTG3P in HCT116 cells. 
(I) CCK8 assay detected the proliferation of HT-29 cells transfected with overexpressed PTTG3P and treated with 2.5mM 2-DG or $100 \mu \mathrm{M} 3$-BP.

(J) Xenograft tumors volume, (K) Xenograft tumors weight were established, with injected with PTTG3P or PTTG3P plus sh-LDHA or PTTG3P treated with 2-DG $(1000 \mathrm{mg} / \mathrm{kg}$, injected into the abdominal cavity). Empty vector as indicated.

(L) Tumor volume and (M) weight were measured in vivo when injected with sh-PTTG3P (20 nmol twice per week) and oxaliplatin treatment ( $5 \mathrm{mg} / \mathrm{kg}$ twice per week, injected into the abdominal cavity) transfected HCT 116 cells.

Data are presented as the mean \pm SD from three independent experiments.

$* \mathrm{P}<0.05,{ }^{*} * \mathrm{P}<0.01, * * * \mathrm{P}<0.001$

\section{Figure 4 PTTG3P regulates Hippo signaling pathway in CRC}

(A) GSEA plot showing that PTTG3P expression positively correlated with YAP-activated gene signatures.

(B) PTTG3P knockdown impaired the mRNA level of YAP1, FOXM1, and CTGF.

(C) PTTG3P knockdown impaired the enrichment of H3K27Ac, H3K27me3 on the promoter of YAP1.

(D) PTTG3P co-expression heat map, TCGA ( https://portal.gdc.cancer.gov/ ) COAD, level 3 HTSeq-FPKM.

(E, F) The effect of HT-29 cells transfected with PTTG3P or PTTG3P+sh-YAP1 or PTTG3P + CA3 (1 $\mu \mathrm{M})$ or PTTG3P+verteporfin $(2 \mu \mathrm{M})$ on cell proliferation by CCK8 assay and apoptosis by flow cytometry assays.

$(\mathrm{G}, \mathrm{H})$ Xenograft tumor volume, Xenograft tumor weight were established, with injected with PTTG3P or PTTG3P+sh-YAP1 or PTTG3P+CA3 $(1.5 \mathrm{mg} / \mathrm{kg})$ or PTTG3P+verteporfin $(50 \mathrm{mg} / \mathrm{kg})$.

(I) CCK8 assay detected the proliferation of HT-29 cells transfected with PTTG3P or PTTG3P plus XMU-MP-1 $(3 \mu \mathrm{M})$

(J) Flow cytometry assays revealed that PTTG3P plus XMU-MP-1 $(3 \mu \mathrm{M})$ could barely rescue cell apoptosis.

Data are presented as the mean $\pm \mathrm{SD}$ from three independent experiments.

$* \mathrm{P}<0.05,{ }^{* *} \mathrm{P}<0.01,{ }^{* * *} \mathrm{P}<0.001$

\section{Figure 5 PTTG3P could induce M2 polarization of macrophage}

(A) RT-PCR was used to detect the expression of M1 markers and M2 markers after LPS/INF- $\gamma$ or IL-4/IL-13 treatment.

(B) The expression of PTTG3P was increased in M2 macrophages.

(C) M2 markers (CD206 and MRC-2) were ablated in the sh-PTTG3P group.

(D) M2 markers (CD206 and MRC-2) were increased in the PTTG3P group.

(E) PTTG3P co-expression heat map, TCGA ( https://portal.gdc.cancer.gov/ ) COAD, level 3 HTSeq-FPKM.

(F)The expression of PTTG3P in HT-29 cells was measured after culturing under normoxia, hypoxia $(1 \% \mathrm{O} 2)$, or $\mathrm{CoCl} 2(100 \mu \mathrm{M})$ for $24 \mathrm{~h}$ by qRT-PCR.

(G)The expression of PTTG3P was evaluated by qRT-PCR in HT-29 cells after knockdown of HIF1A under normoxia condition.

(H)The expression of PTTG3P was evaluated by qRT-PCR in HT-29 cells after knockdown of HIF1A under hypoxia condition. 
(I) PTTG3P correlated with HIF1A.

(J-L) The effect of HT-29 cells transfected with PTTG3P or PTTG3P+si-HIF1A on downstream gene expression, cell proliferation, and apoptosis.

(M) ChIP assay was performed to show HIF1A could directly bind to the PTTG3P promoter region $* \mathrm{P}<0.05,{ }^{*} * \mathrm{P}<0.01,{ }^{* * * \mathrm{P}}<0.001$

\section{Figure 6 PTTG3P regulate in CRC immunology}

(A)The correlation of PTTG3P and immune cell correlation in TCGA-COAD database.

(B) High and low PTTG3P expression with CD8+ T, NK, and TFH cells infiltration in TCGA-COAD database.

(C) Scatter plot of PTTG3P expression and CD8 $+\mathrm{T}$ and NK cells infiltration in TCGA-COAD database.

(D, E) High and low PTTG3P expression with Treg or macrophages infiltration in TCGA-COAD database.

(F) Scatter plot of PTTG3P expression and Treg cells infiltration in TCGA-COAD database.

(G) ELISA detection of the expression of TNF- $\alpha$, IL-1 $\beta$, and IL-6 in cell culture supernatants after depletion of PTTG3P in HCT116 cell.

\section{Figure S1}

(A) According to an online database (GSE84983), the volcano graph showed differential gene.

(B) qRT-PCR of the expressions of the top 5 up-regulated lncRNAs in CRC.

(C) PTTG3P localization was predicted using IncLocator (IncRNA subcellular localization predictor).

(D) PTTG3P localization was validated by subcellular fractionation.

(E-G)Difference in the expression of PTTG3P between CRC samples with somatic mutations in EGFR, VEGFR or RAS and those without such mutations.

(H, I) High YAP1 expression was observed in CRC (TCGA-COAD, $\mathrm{n}=521$ ).

(J) ROC curve analysis of the diagnostic value of YAP1.

$* \mathrm{P}<0.05,{ }^{* *} \mathrm{P}<0.01,{ }^{* * *} \mathrm{P}<0.001$

\section{Figure S2}

Developed radiomics nomogram, the radiomics nomogram was developed with tumor differentiation, tumor size, PTTG3p expression, and YAP1 expression incorporated. 


\section{Figures}

\section{Image not available with this version}

\section{Figure 1}

PTTG3P is upregulated in CRC tissues, and cell lines. (A, B) Exploring PTTG3P expression in CRC cell lines by assembling the Cancer Cell Line Encyclopedia (CCLE) (www.broadinstitute.org/ccle). (C) The expression profiles of PTTG3P in HT-29, SW620, HCT-8, SW480, HCT116, NCM460 and FHC was detected with qRT-PCR. (D, E) qRT-PCR analysis was used to evaluate PTTG3P expression in 120 paired tumor and paired adjacent non-tumor tissues. $(F, G)$ High PTTG3P expression was observed in the TCGA database of CRC $(n=521)$. $(H)$ High PTTG3P expression was observed in the TCGA database of malignant tumors. $(\mathrm{I}, \mathrm{J})$ High PTTG3P expression was observed in STAD and ESCA $(n=20)$. ${ }^{*}<0.05,{ }^{*} \mathrm{P}<0.01,{ }^{* \star *} \mathrm{P}<$ 0.001

\section{Image not available with this version}

\section{Figure 2}

Overexpressed PTTG3P correlates with poor prognosis in CRC. (A) The expression of PTTG3P was checked in different clinical stages of CRC tissues (B) PTTG3P expression and survival predicted poor prognosis of OS in a cohort of 120 paired cases. (C) ROC curve of PTTG3P. (D) Short hairpin RNA (shRNA) targeting PTTG3P and PTTG3P overexpressed plasmids were transfected into HCT116 and HT29 cells. (E) GSEA plot showing that PTTG3P expression positively correlated with glycolysis-activated 
gene signatures (REACTOME GLYCOLYSIS). (F) Analysis of glycolic gene expression in PTTG3P knockdown or re-expressed PTTG3P. (G) qPCR analysis showed that PTTG3P was upregulated under low glucose culture conditions compared to normal glucose in a dose-dependent manner and time-dependent manner. (H) QPCR analysis showed that PTTG3P was increased after 2-DG treatment in dose-dependent manner and in time-dependent manner. ${ }^{*} P<0.05, * * P<0.01$, $* \star * P<0.001$

\section{Image not available with this version}

\section{Figure 3}

PTTG3P promotes the glycolysis and proliferation of CRC. (A) Glucose uptake analysis, (B) Lactate production analysis, (C) ATP analysis explored the glucose uptake, lactate production, and ATP accumulation level, with sh-PTTG3P or overexpressed PTTG3P in HCT116 or HT-29 cells. (D) Extracellular acidification rate (ECAR) analysis tested the glycolytic capacity in CRC cells with sh-PTTG3P or overexpressed PTTG3P in HCT116 or HT-29 cells. (E) CCK8 assay detected the proliferation of HCT116 and HT-29 cells transfected with sh-PTTG3P or overexpressed PTTG3P. (F) Flow cytometry assays revealed that PTTG3P affected cell apoptosis. $(\mathrm{G})$ Tumor volume and $(\mathrm{H})$ weight were measured in vivo when injected with overexpressed PTTG3P in HCT116 cells. (I) CCK8 assay detected the proliferation of HT-29 cells transfected with overexpressed PTTG3P and treated with 2.5mM 2-DG or $100 \mu \mathrm{M}$ 3-BP. (J) Xenograft tumors volume, $(K)$ Xenograft tumors weight were established, with injected with PTTG3P or PTTG3P plus sh-LDHA or PTTG3P treated with 2-DG $(1000 \mathrm{mg} / \mathrm{kg}$, injected into the abdominal cavity). Empty vector as indicated. (L) Tumor volume and $(\mathrm{M})$ weight were measured in vivo when injected with sh-PTTG3P (20 nmol twice per week) and oxaliplatin treatment $₫ 5 \mathrm{mg} / \mathrm{kg}$ twice per week, injected into the

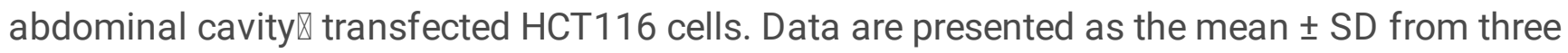
independent experiments. ${ }^{*} \mathrm{P}<0.05,{ }^{*} \mathrm{P}<0.01, * \star \star \mathrm{P}<0.001$ 


\section{Image not available with this version}

\section{Figure 4}

PTTG3P regulates Hippo signaling pathway in CRC (A) GSEA plot showing that PTTG3P expression positively correlated with YAP-activated gene signatures. (B) PTTG3P knockdown impaired the mRNA level of YAP1, FOXM1, and CTGF. (C) PTTG3P knockdown impaired the enrichment of H3K27Ac, H3K27me3 on the promoter of YAP1. (D) PTTG3P co-expression heat map, TCGA ( https://portal.gdc.cancer.gov/ ) COAD, level 3 HTSeq-FPKM. (E, F) The effect of HT-29 cells transfected with PTTG3P or PTTG3P+sh-YAP1 or PTTG3P+CA3 $(1 \mu \mathrm{M})$ or PTTG3P+verteporfin $(2 \mu \mathrm{M})$ on cell proliferation by CCK8 assay and apoptosis by flow cytometry assays. $(G, H)$ Xenograft tumor volume, Xenograft tumor weight were established, with injected with PTTG3P or PTTG3P+sh-YAP1 or PTTG3P+CA3 $(1.5 \mathrm{mg} / \mathrm{kg})$ or PTTG3P+verteporfin $(50 \mathrm{mg} / \mathrm{kg})$. (I) CCK8 assay detected the proliferation of HT-29 cells transfected with PTTG3P or PTTG3P plus XMU-MP-1 $(3 \mu \mathrm{M})(\mathrm{J})$ Flow cytometry assays revealed that PTTG3P plus XMU-MP-1 $(3 \mu \mathrm{M})$ could barely rescue cell apoptosis. Data are presented as the mean $\pm S D$ from three independent experiments. ${ }^{\star} P<0.05,{ }^{\star} * \mathrm{P}<0.01,{ }^{\star} * \star \mathrm{P}<0.001$

\section{Image not available with this version}

\section{Figure 5}

PTTG3P could induce M2 polarization of macrophage (A) RT-PCR was used to detect the expression of M1 markers and M2 markers after LPS/INF- $y$ or IL-4/IL-13 treatment. (B) The expression of PTTG3P was increased in M2 macrophages. (C) M2 markers (CD206 and MRC-2) were ablated in the sh-PTTG3P 
group. (D) M2 markers (CD206 and MRC-2) were increased in the PTTG3P group. (E) PTTG3P coexpression heat map, TCGA ( https://portal.gdc.cancer.gov/ ) COAD, level 3 HTSeq-FPKM. (F)The expression of PTTG3P in HT-29 cells was measured after culturing under normoxia, hypoxia ( $1 \%$ O2), or $\mathrm{CoCl} 2(100 \mu \mathrm{M})$ for $24 \mathrm{~h}$ by qRT-PCR. (G)The expression of PTTG3P was evaluated by qRT-PCR in HT-29 cells after knockdown of HIF1A under normoxia condition. (H)The expression of PTTG3P was evaluated by qRT-PCR in HT-29 cells after knockdown of HIF1A under hypoxia condition. (I) PTTG3P correlated with HIF1A. (J-L) The effect of HT-29 cells transfected with PTTG3P or PTTG3P+si-HIF1A on downstream gene expression, cell proliferation, and apoptosis. (M) ChIP assay was performed to show HIF1A could directly bind to the PTTG3P promoter region $* P<0.05$, $* * P<0.01$, ${ }^{\star \star *} \mathrm{P}<0.001$

\section{Image not available with this version}

\section{Figure 6}

PTTG3P regulate in CRC immunology (A)The correlation of PTTG3P and immune cell correlation in TCGA-COAD database. (B) High and low PTTG3P expression with CD8+ T, NK, and TFH cells infiltration in TCGA-COAD database. (C) Scatter plot of PTTG3P expression and CD8+ T and NK cells infiltration in TCGA-COAD database. (D, E) High and low PTTG3P expression with Treg or macrophages infiltration in TCGA-COAD database. (F) Scatter plot of PTTG3P expression and Treg cells infiltration in TCGA-COAD database. (G) ELISA detection of the expression of TNF- $a$, IL-1 $1 \beta$, and IL- 6 in cell culture supernatants after depletion of PTTG3P in HCT116 cell.

\section{Supplementary Files}

This is a list of supplementary files associated with this preprint. Click to download.

- figures1.tif

- figureS2Nomogram.tif

- supplementarytable.doc 